\title{
PERCUTANEOUS NEPHROLITOTOMY IN PEDIATRIC PATIENTS
}

\author{
M.R. Darabi Mahboub ${ }^{1}$, R. Taghavi ${ }^{2}$ \\ ${ }^{1}$ Urology, ${ }^{2}$ Mashhad University of Medical Science, Mashhad, Iran
}

Introduction: Percutaneous nephrolithotomy is a safe procedure in pediatric and adult group for renal stone. We reported my experience PCNL in pediatric group in our department.

Methods: Percutaneous nephrolitotomy (PNL) was performed in 25 children in the age range of 4-12 years with the diagnosis of renal stones. 11 of them anderogen ESWL before PCNL which it was not successful. Necessary lab test and IVP were done in all patients. 16 patients had single pelvic stone. 9 patients had middle and inferior calcyeal stones other than pelvic stones. 12 of patients were female $(50 \%)$ and 13 of them male. 3 patient had under gone previous operation on the same kidney.

Results: We could access to the kidney in all 25 patients, after nephroscopy we removed the single pelvic stones in 4 patients. In other 21 patients we first broke stones with ultrasonic or pneumatic lithoclast and then remove them. In patient we couldn't remove the middle calyceal stone. 22 patients become stones free. 7 patients had stones in the middle calyx. We had bleeding in 6 patients and fever in 5 patients which improved treatment.

Conclusion: Our experience in PCNL of adult and pediatric patients shows that PCNL is a save and effective methods with alitte complication in adults and pediatrics. We recommend PCNL in children. 